\title{
Application of DFTB and Machine Learning
}

\section{to Evaluate the Stability of Biomass}

\section{Intermediates on the Rh(111) Surface}

\author{
Chaoyi Chang and Andrew J. Medford*
}

School of Chemical 83 Biomolecular Engineering, Georgia Institute of Technology, 30332

\author{
E-mail: ajm@gatech.edu
}

\section{Supporting Information Available}

SMILES string generation for adsorbates

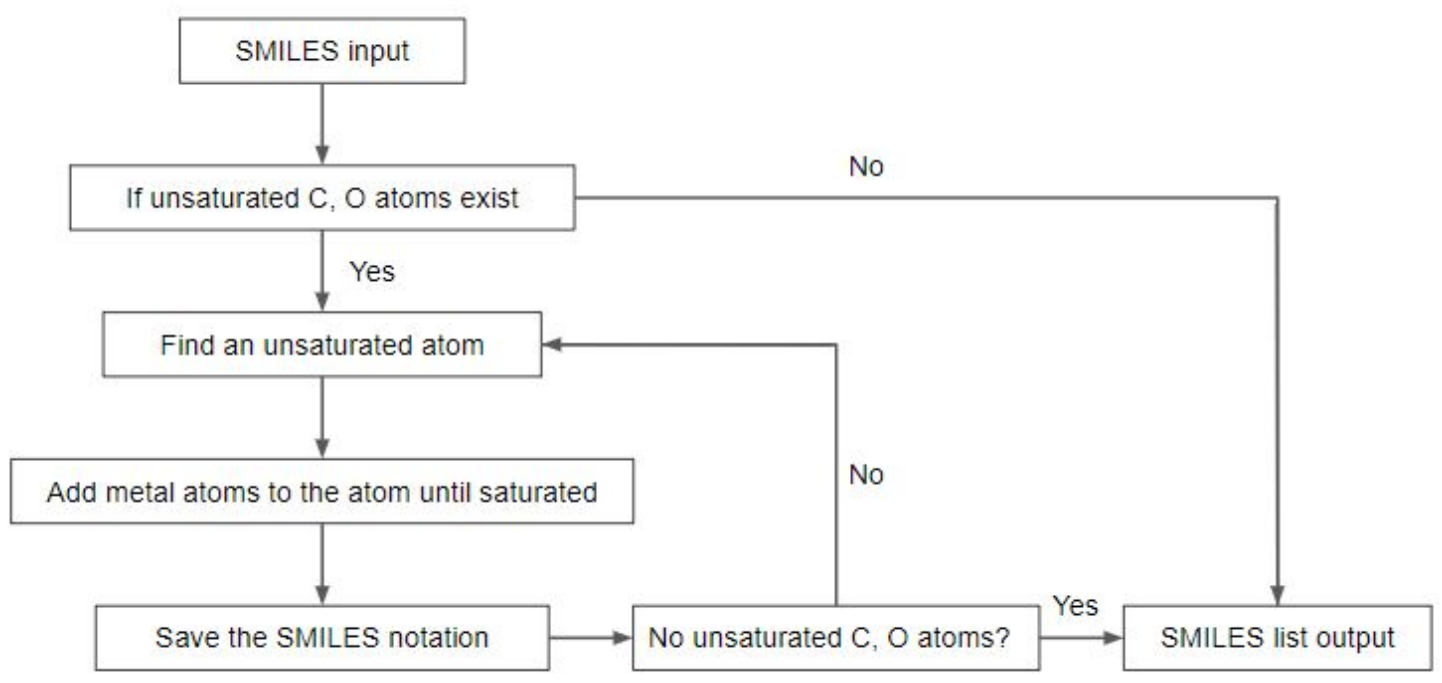

Figure S1: Generating algorithms of SMILES notation of adsorbate with metal atoms 


\section{Reaction types identified from vector clustering}

Table S1: 83 clusters from R0 vectors with atomic environment (\# of hydrogen atom and heavy atom surrounding)

\begin{tabular}{|c|c|c|c|c|c|}
\hline cluster & hydrogen_0 & heavy_atom_0 & hydrogen_1 & heavy_atom_1 & label \\
\hline 1 & 2 & 3 & 1 & 4 & $\mathrm{C}-\mathrm{C}$ \\
\hline 2 & 2 & 3 & 0 & 4 & $\mathrm{C}-\mathrm{C}$ \\
\hline 3 & 0 & 4 & 0 & 3 & $\mathrm{C}-\mathrm{C}$ \\
\hline 4 & 1 & 4 & 1 & 3 & $\mathrm{C}-\mathrm{C}$ \\
\hline 5 & 1 & 3 & 0 & 3 & C-C \\
\hline 6 & 0 & 4 & 1 & 3 & $\mathrm{C}-\mathrm{C}$ \\
\hline 7 & 2 & 3 & 1 & 3 & $\mathrm{C}-\mathrm{C}$ \\
\hline 8 & 1 & 4 & 0 & 3 & $\mathrm{C}-\mathrm{C}$ \\
\hline 9 & 0 & 3 & 2 & 3 & $\mathrm{C}-\mathrm{C}$ \\
\hline 10 & 1 & 3 & 1 & 3 & $\mathrm{C}-\mathrm{C}$ \\
\hline 11 & 0 & 3 & 0 & 3 & $\mathrm{C}-\mathrm{C}$ \\
\hline 12 & 0 & 4 & 0 & 4 & $\mathrm{C}-\mathrm{C}$ \\
\hline 13 & 2 & 3 & 2 & 3 & $\mathrm{C}-\mathrm{C}$ \\
\hline 14 & 1 & 4 & 0 & 4 & $\mathrm{C}-\mathrm{C}$ \\
\hline 15 & 1 & 4 & 1 & 4 & $\mathrm{C}-\mathrm{C}$ \\
\hline 16 & 0 & 2 & 1 & 4 & $\mathrm{C}-\mathrm{C}$ \\
\hline 17 & 0 & 2 & 1 & 3 & $\mathrm{C}-\mathrm{C}$ \\
\hline 18 & 0 & 2 & 2 & 3 & $\mathrm{C}-\mathrm{C}$ \\
\hline 19 & 0 & 2 & 0 & 4 & $\mathrm{C}-\mathrm{C}$ \\
\hline 20 & 0 & 2 & 0 & 3 & $\mathrm{C}-\mathrm{C}$ \\
\hline 21 & 0 & 2 & 0 & 2 & C-C \\
\hline 22 & 1 & 2 & 2 & 2 & C-C \\
\hline
\end{tabular}


Table S1 - continued from previous page

\begin{tabular}{|c|c|c|c|c|c|}
\hline cluster & hydrogen_0 & heavy_atom_0 & hydrogen_1 & heavy_atom_1 & label \\
\hline 23 & 2 & 2 & 1 & 4 & C-C \\
\hline 24 & 2 & 2 & 0 & 3 & $\mathrm{C}-\mathrm{C}$ \\
\hline 25 & 0 & 4 & 2 & 2 & $\mathrm{C}-\mathrm{C}$ \\
\hline 26 & 2 & 2 & 1 & 3 & C-C \\
\hline 27 & 2 & 2 & 2 & 3 & $\mathrm{C}-\mathrm{C}$ \\
\hline 28 & 0 & 2 & 2 & 2 & $\mathrm{C}-\mathrm{C}$ \\
\hline 29 & 2 & 2 & 2 & 2 & $\mathrm{C}-\mathrm{C}$ \\
\hline 30 & 0 & 2 & 1 & 2 & $\mathrm{C}-\mathrm{C}$ \\
\hline 31 & 1 & 2 & 1 & 4 & $\mathrm{C}-\mathrm{C}$ \\
\hline 32 & 1 & 2 & 0 & 4 & $\mathrm{C}-\mathrm{C}$ \\
\hline 33 & 1 & 2 & 1 & 3 & $\mathrm{C}-\mathrm{C}$ \\
\hline 34 & 1 & 2 & 0 & 3 & $\mathrm{C}-\mathrm{C}$ \\
\hline 35 & 1 & 2 & 2 & 3 & $\mathrm{C}-\mathrm{C}$ \\
\hline 36 & 1 & 2 & 1 & 2 & $\mathrm{C}-\mathrm{C}$ \\
\hline 37 & 3 & 2 & 0 & 4 & $\mathrm{C}-\mathrm{C}$ \\
\hline 38 & 3 & 2 & 0 & 3 & $\mathrm{C}-\mathrm{C}$ \\
\hline 39 & 3 & 2 & 2 & 3 & $\mathrm{C}-\mathrm{C}$ \\
\hline 40 & 1 & 3 & 3 & 2 & $\mathrm{C}-\mathrm{C}$ \\
\hline 41 & 3 & 2 & 0 & 2 & C-C \\
\hline 42 & 2 & 2 & 3 & 2 & $\mathrm{C}-\mathrm{C}$ \\
\hline 43 & 1 & 2 & 3 & 2 & C-C \\
\hline 44 & 1 & 4 & 1 & 2 & $\mathrm{C}-\mathrm{O}$ \\
\hline 45 & 1 & 3 & 1 & 2 & $\mathrm{C}-\mathrm{O}$ \\
\hline 46 & 0 & 4 & 1 & 2 & $\mathrm{C}-\mathrm{O}$ \\
\hline
\end{tabular}


Table S1 - continued from previous page

\begin{tabular}{|c|c|c|c|c|c|}
\hline cluster & hydrogen_0 & heavy_atom_0 & hydrogen_1 & heavy_atom_1 & label \\
\hline 47 & 0 & 3 & 1 & 2 & C-O \\
\hline 48 & 2 & 3 & 1 & 2 & $\mathrm{C}-\mathrm{O}$ \\
\hline 49 & 2 & 2 & 1 & 2 & $\mathrm{C}-\mathrm{O}$ \\
\hline 50 & 0 & 2 & 1 & 2 & $\mathrm{C}-\mathrm{O}$ \\
\hline 51 & 2 & 2 & 0 & 2 & $\mathrm{C}-\mathrm{O}$ \\
\hline 52 & 1 & 3 & 0 & 2 & $\mathrm{C}-\mathrm{O}$ \\
\hline 53 & 1 & 4 & 0 & 2 & $\mathrm{C}-\mathrm{O}$ \\
\hline 54 & 0 & 3 & 0 & 2 & $\mathrm{C}-\mathrm{O}$ \\
\hline 55 & 0 & 4 & 0 & 2 & $\mathrm{C}-\mathrm{O}$ \\
\hline 56 & 2 & 3 & 0 & 2 & $\mathrm{C}-\mathrm{O}$ \\
\hline 57 & 1 & 2 & 1 & 2 & $\mathrm{C}-\mathrm{O}$ \\
\hline 58 & 1 & 2 & 0 & 2 & $\mathrm{C}-\mathrm{O}$ \\
\hline 59 & 0 & 2 & 0 & 2 & $\mathrm{C}-\mathrm{O}$ \\
\hline 60 & 3 & 1 & 1 & 1 & $\mathrm{C}-\mathrm{H}$ \\
\hline 61 & 3 & 2 & 1 & 1 & $\mathrm{C}-\mathrm{H}$ \\
\hline 62 & 1 & 1 & 1 & 1 & $\mathrm{C}-\mathrm{H}$ \\
\hline 63 & 1 & 4 & 1 & 1 & $\mathrm{C}-\mathrm{H}$ \\
\hline 64 & 1 & 3 & 1 & 1 & $\mathrm{C}-\mathrm{H}$ \\
\hline 65 & 1 & 2 & 1 & 1 & C-H \\
\hline 66 & 2 & 2 & 1 & 1 & $\mathrm{C}-\mathrm{H}$ \\
\hline 67 & 2 & 3 & 1 & 1 & $\mathrm{C}-\mathrm{H}$ \\
\hline 68 & 2 & 1 & 1 & 1 & C-H \\
\hline 69 & 1 & 2 & 1 & 1 & $\mathrm{O}-\mathrm{H}$ \\
\hline 70 & 1 & 1 & 1 & 1 & $\mathrm{O}-\mathrm{H}$ \\
\hline
\end{tabular}


Table S1 - continued from previous page

\begin{tabular}{|l|l|l|l|l|l|}
\hline cluster & hydrogen_0 & heavy_atom_0 & hydrogen_1 & heavy_atom_1 & label \\
\hline 71 & 2 & 3 & & & C-M \\
72 & 1 & 4 & & C-M \\
73 & 0 & 5 & & C-M \\
74 & 0 & 3 & & C-M \\
75 & 1 & 3 & & C-M \\
76 & 0 & 2 & & C-M \\
77 & 2 & 2 & & C-M \\
78 & 3 & 2 & & \\
79 & 0 & 3 & & & C-M \\
80 & 1 & 2 & & & C-M \\
81 & 0 & 2 & & & O-M \\
82 & 0 & 1 & & & \\
83 & 1 & & & & \\
\hline
\end{tabular}

\section{Confidence Interval Calculation}

The $75 \%$ confidence of the $0.45 \mathrm{eV}$ criterion is calculated as following (assuming $\hat{E}_{1}-\hat{E}_{2}>$ 0.45 and $\sigma=0.45 \mathrm{eV})$ : 


$$
\begin{aligned}
& E-\hat{E}=Z \sim \operatorname{Normal}\left(0, \sigma^{2}\right) \\
& E_{1}=\hat{E}_{1}+Z \sim \operatorname{Normal}\left(\hat{E}_{1}, \sigma^{2}\right) \\
& E_{2}=\hat{E}_{2}+Z \sim \operatorname{Normal}\left(\hat{E}_{2}, \sigma^{2}\right) \\
& E_{1} \Perp E_{2} \Rightarrow E_{1}-E_{2} \sim \operatorname{Normal}\left(\hat{E}_{1}-\hat{E}_{2}, 2 \sigma^{2}\right) \\
& P\left(E_{1}-E_{2}<0\right)=\Phi\left(\frac{\hat{E}_{2}-\hat{E}_{1}}{2 \sigma^{2}}\right)=1-\Phi\left(\frac{\hat{E}_{1}-\hat{E}_{2}}{2 \sigma^{2}}\right) \\
& \frac{\hat{E}_{1}-\hat{E}_{2}}{\sqrt{2} \sigma}>\frac{1}{\sqrt{2}} \Rightarrow P\left(E_{1}-E_{2}<0\right)<1-\Phi\left(\frac{1}{\sqrt{2}}\right)=0.25 \\
& P\left(E_{1}<E_{2} \mid \hat{E}_{1}-\hat{E}_{2}>0.45\right)<0.25 \\
& \Rightarrow P\left(E_{1}>E_{2} \mid \hat{E}_{1}-\hat{E}_{2}>0.45\right)>0.75
\end{aligned}
$$

The following files are available free of charge via the zip file in the supplementary information or via GitHub: https://github.com/medford-group/Rh_biomass_ML.

- regression: contains OLS, PLS and LDA regression scripts and the DFT regression data in .json file

- all_adsorbates.smi: contains SMILES notation of all adsorbates used in this study

- model: contains the Mol2Vec training data (groups.smi) and the Mol2Vec corpus data (groups.cp)

- spearman: contains Spearman's correlation coefficient calculation script and the DFT, PLS and LDA regression data in .json file

- dft_diff.json: contains SMILES notations of previous lowest energy geometry, new lowest energy geometry and energy difference for the adsorbates found by ML models

- hotbit_params: contains parameters used for DFTB model. 